\title{
Structural, Elastic and Thermodynamic Properties of ScP Compound: DFT Study
}

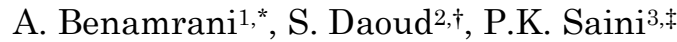 \\ ${ }^{1}$ Département des Sciences et Techniques, Faculté des Sciences et de la Technologie, Université Mohamed Elbachir \\ El Ibrahimi de Bordj Bou Arreridj, Bordj Bou Arreridj, 34000, Algérie \\ ${ }^{2}$ Laboratoire Matériaux et Systèmes Electroniques, Faculté des Sciences et de la Technologie, Université Mohamed \\ Elbachir El Ibrahimi de Bordj Bou Arreridj, Bordj Bou Arreridj, 34000, Algérie \\ ${ }^{3}$ Department of Physics, Government College, Hansi, Haryana, 125033, India
}

(Received 17 January 2021; revised manuscript received 23 February 2021; published online 25 February 2021)

\begin{abstract}
The present work aims to investigate the structural parameters, elastic constants and thermodynamic properties of scandium mono-phosphide ( $\mathrm{ScP}$ ) binary compound. We employed the Quantum Espresso code with the projected augmented wave (PAW) pseudopotentials approach in the framework of the density functional theory (DFT). Firstly, we report the ground state parameters of both NaCl-type (B1) and CsCltype (B2) phases. Generally, our obtained data agree well with other results of the literature. Our calculated value (5.205 $\AA$ ) of $a_{0}$ for B1 phase deviates from the experimental result (5.312 $\AA$ ) by around $2 \%$.

The structural phase transition from B1 to B2 phase under pressure was determined. Our calculation shows that ScP transforms from B1 to B2 phase at a pressure of $108 \mathrm{GPa}$, with a volume collapse of $4.3 \%$.

Secondly, we calculate the elastic stiffness constants, elastic moduli, anisotropy factor, Poisson's ratio, Vickers hardness, fracture toughness, acoustic wave speeds, Debye temperature and melting point of the most stable phase (B1). Our data of the elastic constants are also in good agreement with other theoretical results of the literature. The Vickers hardness $H_{V}$ of $\mathrm{ScP}$ compound was found equal to $12.7 \mathrm{GPa}$, while the Debye temperature $\theta_{D}$ was found at around 551.8 (522.1) K. Moreover, some interesting thermodynamic properties, especially the energy, free energy, entropy and constant volume heat capacity as a function of temperature were also reported and discussed.
\end{abstract}

Keywords: ScP material, Phase transition, Elastic constant, Hardness, Thermodynamic properties.

DOI: $10.21272 /$ jnep.13(1).01008

PACS numbers: 62.20.de, 62.30. + d, 64.70.kd, 65.40. $+\mathrm{g}$

\section{INTRODUCTION}

Scandium III-V based materials have recently received more attention due to having their potential applications in high power devices. There have been numerous papers [1-5] published so far on the structural and electronic properties of these materials. Instead, only very experimental results [2] will be reported.

Using the first-principle Savrasov version of the full potential linear muffin-tin orbitals (FP-LMTO) method, Tebboune et al. [1] investigated the structural phase transition under high pressure of $\mathrm{ScN}, \mathrm{ScP}, \mathrm{ScAs}$ and $\mathrm{ScSb}$ binary compounds. At normal conditions, the ground-state configuration of these materials is the NaCl-type (B1) structure [1]. Moreover, based on the curves energy-volume $(E-V)$, Tebboune et al. [1] mentioned that the $\mathrm{B} 1$ phase of all these materials transforms to CsCl-type (B2) phase under high pressure.

Parthé and Parthé [2] have determined experimentally the lattice parameters of both $\mathrm{ScP}$ and yttrium phosphide (YP) materials using the X-ray technique. They found that both $\mathrm{ScP}$ and YP crystallize in the B1 structure, with lattice constants of around $5.312 \AA$ for ScP and 5.661 A for YP, respectively.

Benalia et al. [3] investigated the structural and electronic properties of both $\mathrm{Sc}_{x} \mathrm{Ga}_{1-x} \mathrm{P}$ and $\mathrm{Sc}_{x} \mathrm{Al}_{1-x} \mathrm{P}$ ternary alloys using the FP-LMTO approach, while Celin-Mancera and co-authors [4] studied the electronic band structures, elastic constants, and thermodynamic properties of $\mathrm{Sc}_{x} \mathrm{Ga}_{1-x} \mathrm{P}(x=25,50$, and $75 \%)$ ternary alloys in both ZnS-type and NaCl-type phases. Their results show that the most stable phase is the B1 for both $\mathrm{Sc}_{0.5} \mathrm{Ga} 0.5 \mathrm{P}$ and $\mathrm{Sc}_{0.75} \mathrm{Ga} 0.25 \mathrm{P}$, while the $\mathrm{B} 3$ was found for $\mathrm{Sc}_{0.25} \mathrm{Ga}{ }_{0.75} \mathrm{P}$ ternary alloy.

Karil et al. [5] studied the effect of pressure on the structural phase transition and elastic constants of ScP compound using an effective inter-ionic potential method. This material has an indirect band gap, with energy of around $1.1 \mathrm{eV}$ at room temperature, and it is usually used in high frequency applications, laser diodes fabrication, and high power devices [5]. Increasing pressure gradually, Karil and co-authors [5] found that all the elastic constants $C_{i j}$ of $\mathrm{ScP}$ compound increase monotonically. They found that the $\mathrm{B} 1$ phase of this material transforms to B2 phase at pressure of $250 \mathrm{GPa}$, with a very small volume collapse of $1.4 \%$.

In the present work, we report on first-principles calculations of the equation of state (EoS) parameters and elastic properties of ScP using the the Quantum Espresso (QE) software [6] and the complementary package Thermo_pw [7]. Some other interesting thermodynamic properties such as the vibrational energy, vibrational free energy, entropy, and the heat capacity as a function of temperature of ScP compound are also reported and analyzed.

\footnotetext{
*quantum.ammar@gmail.com

$\dagger$ salah_daoud07@yahoo.fr

‡pawansaini2005@gmail.com
} 


\section{COMPUTATIONAL METHOD}

In this work, we used the Quantum Espresso code [6], based on the projected augmented wave (PAW) method and a plane waves basis set with pseudopotentials (P.pz-n-kjpaw_psl.0.1.UPF, for Phosphor, and Sc.pz-spn-kjpaw_psl.1.0.0.UPF, for Scandium) [8]. The exchange-correlation potential was treated using the local density approximation (LDA) [9]. The wave functions were expanded with an energy cut-off $=70$ Ry for the plane wave basis set. The charge density cut-off was chosen $=560 \mathrm{Ry}$, which are largely sufficient to achieve a convergence with an energy threshold of $1 \times 10^{-5} \mathrm{Ry}$, and the forces are small than $1 \times 10^{-4} \mathrm{Ry} / \mathrm{Bohr}$. The integration over the Brillouin zone was performed using $4 \times 4 \times 4 \quad k$-points Monkhorst-Pack [10] mesh and a Gaussian smearing scheme has been used with 0.03 Ry width. The elastic constants and the thermodynamic properties have been performed using Thermo_PW package [7]. The theory related with the package thermo_pw [7] have not been explained here in detail, however the reader can find more details and formulas implemented within thermo_pw package to calculate different elastic and thermodynamic properties (please see: M. Palumbo, A. Dal Corso, J. Phys. :Condens. Matter, 29 (2017) 395401; C. Malika, A. Dal Corso, J. Phys.: Condens. Matter, 32 (2020) 315902; C. Malika, A. Dal Corso, J. Appl. Phys., 127 (2020) 245103).

\section{DISCUSSION OF RESULTS}

\subsection{Equation of State Parameters}

In order to determine the equation of state (EoS) parameters of $\mathrm{ScP}$ compound, the total energy versus volume $(E-V)$ data was used. The equilibrium lattice constant $a_{0}$, the bulk modulus $B_{0}$, and the pressure derivative of the bulk modulus $B_{0}{ }^{\prime}$ have been determined using the Murnaghan's EoS [11]. Fig. 1 shows the variation of the total energy $E$ of both B1 and B2 phases as a function of the volume $V$.

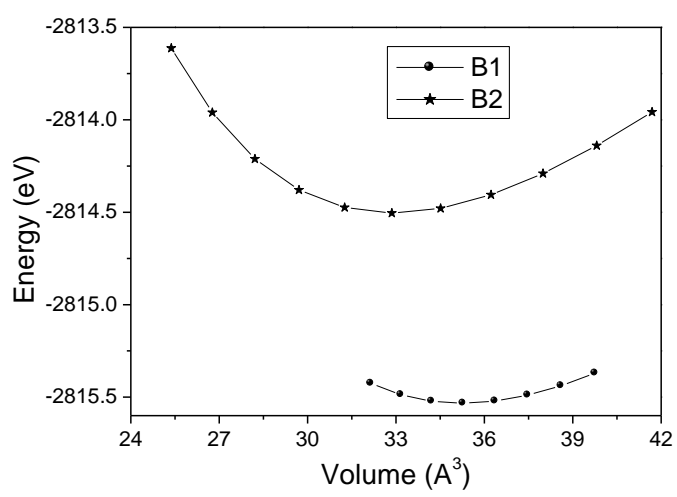

Fig. 1 - Variations of the total energy for ScP in both $\mathrm{B} 1$ and B2 phases as a function of volume

It can be seen from $E$ - $V$ curves, that the most stable phase of $\mathrm{ScP}$ at normal conditions is the $\mathrm{B} 1$ phase since the lowest energy at equilibrium corresponds to B1-ScP, which is consistent well with the experimental and other theoretical works $[2,13]$. The calculated values of $a_{0}, B_{0}$, and $B_{0}{ }^{\prime}$ with both $\mathrm{B} 1$ and $\mathrm{B} 2$ phases are listed in Table 1, and compared to the experimental data [2], and the results of other theoretical approaches [1, 4, 12-14]. From Table 1, one can note that our calculated value $(5.205 \AA)$ of $a_{0}$ for $\mathrm{B} 1$ phase is in general agreement with the experimental result (5.312 $\AA$ ) reported in Ref. [2]. The deviations between our value of $a_{0}$ and the experimental one reported by Parthé and Parthé [2] is only around $2 \%$. Our calculated result $(109.5 \mathrm{GPa})$ of $B_{0}$ for B1 phase is also in good agreement with the theoretical value $(107.9 \mathrm{GPa})$ reported by Celin-Mancera and co-authors [4]. The deviation between our value of $B_{0}$ and the theoretical value $(107.9 \mathrm{GPa})$ reported by Celin-Mancera et al. [1] is only around $1.5 \%$.

As far as $a_{0}$ and $B_{0}$ are concerned, our calculated values appear to be closer to that reported by CelinMancera et al. [4] using the LDA approach than those published by Maachou et al. [13] using the GGA approach. This is an expected result since in the present work where the exchange-correlation potential has been calculated within the LDA approach

Table 1 - EOS parameters of ScP material in both B1 and B2 phases, in comparison with other data of the literature, a) - Ref. [1], b) - Ref. [2] Exp, c) - Ref. [4] LDA, d) - Ref. [4] GGA,-e) - Ref. [4] WC,f) - Ref. [12] GGA, g) - Ref. [13] GGA, h) - Ref. [14] GGA

\begin{tabular}{|c|c|c|c|}
\hline Phase & Parameter & This work & Other works \\
\hline \multirow{3}{*}{ B1 } & $a_{0}(\AA)$ & 5.205 & $\begin{array}{l}5.354^{\mathrm{a}}, 5.312^{\mathrm{b}}, 5.204^{\mathrm{c}}, \\
5.319^{\mathrm{d}}, 5.2584^{\mathrm{e}}, 5.31^{\mathrm{f}} \\
5.32^{\mathrm{g}}, 5.27^{\mathrm{h}}\end{array}$ \\
\hline & $B_{0}(\mathrm{GPa})$ & 109.5 & $\begin{array}{l}111.07^{\mathrm{a}}, 107.9^{\mathrm{c}}, 94.9^{\mathrm{d}} \text {, } \\
101.4^{\mathrm{e}}, 97.6^{\mathrm{f}}, 99.27^{\mathrm{g}} \\
110.05^{\mathrm{h}}\end{array}$ \\
\hline & $B_{0}^{\prime}$ & 3.77 & $\begin{array}{l}2.903^{\mathrm{a}}, 3.41^{\mathrm{f}}, 3.58^{\mathrm{g}}, \\
5.80^{\mathrm{h}}\end{array}$ \\
\hline \multirow{3}{*}{ B2 } & $a_{0}(\AA)$ & 3.205 & $3.338^{\mathrm{a}}, 3.27^{\mathrm{f}}, 3.27^{\mathrm{g}}$ \\
\hline & $B_{0}(\mathrm{GPa})$ & 107.58 & $112.68^{\mathrm{a}}, 97.1^{\mathrm{f}}, 101.82^{\mathrm{g}}$ \\
\hline & $B_{0}^{\prime}$ & 3.76 & $2.849^{\mathrm{a}}, 4.4^{\mathrm{f}}, 3.66^{\mathrm{g}}$ \\
\hline
\end{tabular}

\subsection{Structural Phase Transition}

Since the different quantities are calculated at $T=0 \mathrm{~K}$, so the most stable phase at any pressure can be obtained using the enthalpy $H$, which is expressed as follows: $H=E+P V$ [12]. The transition pressure $\left(P_{t}\right)$ from the most stable phase $(\mathrm{NaCl})$ to $\mathrm{CsCl}$ structure was calculated using the $H$ as a function of the pressure. The results obtained are plotted in Fig. 2. It may be expected that the enthalpies $H$ of both $\mathrm{B} 1$ and $\mathrm{B} 2$ phases show a linear increase as the pressure increases.

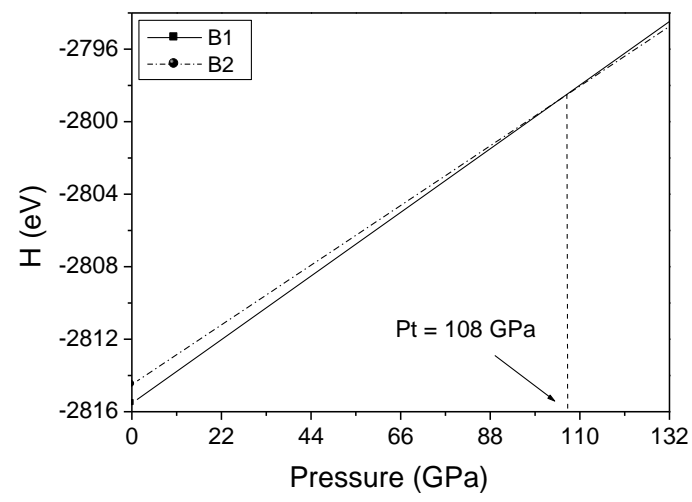

Fig. 2 - Enthalpies of both B1 and B2 phases versus pressure 
Our calculated value of the transition pressure $\left(P_{t}\right)$ was reported in Table 2, along other theoretical data [1, $5,12,13]$. From Table 2, one can observe that our value (108 GPa) of $P_{t}$ is very lower than the result (330 GPa) reported in Ref. [1]. It is also slightly lower than other theoretical values: 250, 247.56, and $245.61 \mathrm{GPa}$ reported by Karil and co-authors [5], Pandit et al. [12], and Maachou et al. [13], respectively.

Table 2 - Calculated transition pressure $P_{t}$ and volume collapse $V_{t}$ for $\mathrm{ScP}$ compound, in comparison with other theoretical data. a) - Ref. [1], b) - Ref. [5], c) - Ref. [12], d) - Ref. [13]

\begin{tabular}{|c|c|c|}
\hline Parameter & $P_{t}(\mathrm{GPa})$ & $V_{t}(\%)$ \\
\hline This work & 108 & 4.3 \\
\hline Other works & $330^{\mathrm{a}}, 250^{\mathrm{b}}, 247.56^{\mathrm{c}}, 245.61^{\mathrm{d}}$ & $1.4^{\mathrm{b}}$ \\
\hline
\end{tabular}

We have calculated the relative volume $V(P) / V_{0}$ at different pressures ( $V_{0}$ is the conventional volume at $P=0 \mathrm{Gpa})$. The variations of the volume versus applied pressure $\mathrm{P}-\left(V p / V_{0}\right)$ curves of $\mathrm{ScP}$ compound for $\mathrm{NaCl}$ $\rightarrow \mathrm{CsCl}$ transition were plotted in Fig. 3 .

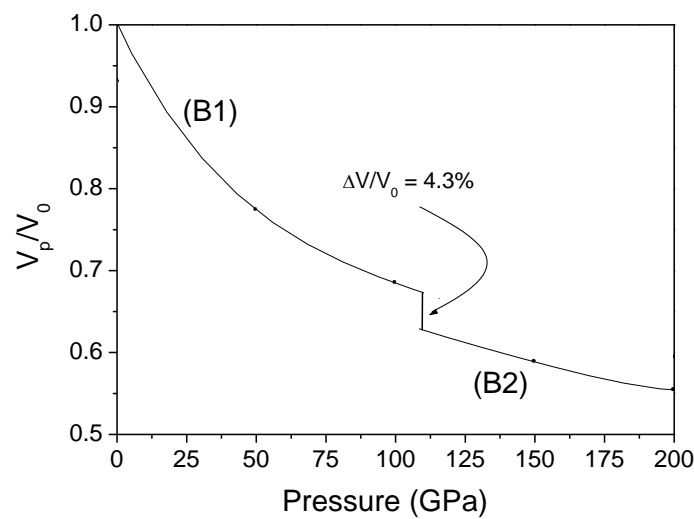

Fig. 3 - Variations of the relative volume $V(P) / V_{0}$ as a function of pressure for both $\mathrm{B} 1$ and $\mathrm{B} 2$ phases

As shown in Fig. 3, the behavior of the volume change is usual with pressure. From the previous curves, one can predict the volume collapse [- $\left(V_{\mathrm{B} 2(P t)}-\right.$ $\left.\left.\left.V_{\mathrm{B} 1(P t))}\right) / V_{0}\right)\right]=\left(-\Delta V / V_{0}\right)$ at the point of transition. It can be seen that there is about $4.3 \%$ volume reduction in $\mathrm{B} 1 \rightarrow \mathrm{B} 2$ phase transition for $\mathrm{ScP}$. It is very clear that our result (4.3\%) regarding the volume collapse during the transition from $\mathrm{B} 1 \rightarrow \mathrm{B} 2$ is quite different than the theoretical value (1.4\%) reported by Karil et al. [5].

\subsection{Elastic Constants}

Elastic constants have been calculated through second derivative of Helmholtz free energy versus strain as follows:

$$
\tilde{C}_{i j k l}=\frac{1}{V}\left(\frac{\partial^{2} F}{\partial \epsilon_{i j} \partial \epsilon}\right)_{\epsilon=0},
$$

where $F$ is the Helmholtz free energy, which is the sum of DFT total energy and the vibrational free energy, $V$ is the volume of unit cell, $\epsilon$ is the strain (for more details, please see for example: C. Malika, A. Dal Corso, J. Phys.: Condens. Matter, 32 (2020) 315902).

In cubic crystal, there are only three independent elastic constants $\left(C_{11}, C_{12}\right.$, and $\left.C_{44}\right)$. Our calculated results on the $C_{i j}$ of $\mathrm{ScP}$ are listed in Table 2, along with other theoretical data [12, 14-16]. The mechanical stability of ScP material is checked in this study in terms of the elastic constants $C_{i j}$ using the zeropressure elastic stability criteria of cubic crystals [4]

$$
\mathrm{C}_{11}>0 ; \mathrm{C}_{44}>0 ; \mathrm{C}_{11}>\left|\mathrm{C}_{12}\right| ;\left(\mathrm{C}_{11}+2 \mathrm{C}_{12}\right)>0 \text {. }
$$

Based on our data, all criteria mentioned in Eq. (2) are satisfied, which indicates that the $\mathrm{ScP}$ material in cubic NaCl-type is mechanically stable at equilibrium. From Table 3, one can observe that our calculated values of the elastic constants $C_{i j}$ are in general agreement with the theoretical results [12,14-16]. For example, the deviation between our value $(37.44 \mathrm{GPa})$ of $C_{12}$ and the value $(37.36 \mathrm{GPa})$ reported by Shoaib et al. [14] is only around $0.2 \%$, while the difference between our value (49.24 GPa) of $C_{44}$ and the theoretical data (49.32 GPa) reported by Kube [16] is only around $0.16 \%$.

Table 3 - Calculated elastic constants $C_{i j}$, bulk modulus $B$ and elastic anisotropy $A$ for (B1) ScP compound, in comparison with other theoretical data of the literature, a) - Ref. [12], b) - Ref. [14], c) - Ref. [15] , d) - Ref. [16]

\begin{tabular}{|c|l|l|}
\hline Parameter & This work & \multicolumn{1}{c|}{ Other works } \\
\hline$C_{11}(\mathrm{GPa})$ & 252.79 & $202^{\mathrm{a}}, 225.76^{\mathrm{b}}, 216.53^{\mathrm{c}, \mathrm{d}}$ \\
\hline$C_{12}(\mathrm{GPa})$ & 37.44 & $46^{\mathrm{a}}, 37.36^{\mathrm{b}}, 38.10^{\mathrm{c}, \mathrm{d}}$ \\
\hline$C_{44}(\mathrm{GPa})$ & 49.24 & $87^{\mathrm{a}}, 40.84^{\mathrm{b}}, 49.32^{\mathrm{c}, \mathrm{d}}$ \\
\hline
\end{tabular}

To the best of our knowledge, no measurements of the elastic constants $C_{i j}$ for $\mathrm{ScP}$ compound have been reported; however the crystal of $\mathrm{ScP}$ in cubic NaCl-type has been elaborated since 1965 [2].

The bulk modulus $B$ and the anisotropic index $A$ are derived from the elastic constants $C_{i j}$ as follows [17]:

$$
B=\left(C_{11}+2 C_{12}\right) / 3 \text {, and } A=\left(2 C_{44}\right) /\left(C_{11}-C_{12}\right) .
$$

Our results of $B$ and $A$ for $\mathrm{ScP}$ material were found to be $109.2 \mathrm{GPa}$ and 0.46 , respectively. Our calculated result of $B$ is very well agreed with the value (109.5 GPa) obtained from the fitting of $E-V$ data. The deviation between the two values is only around $0.3 \%$. In other hand, our value (0.46) of $A$ is slightly higher than the theoretical values 0.43 reported by Shoaib et al. [14], and slightly lower than the value (0.55) of Kube [16].

The Young modulus $E$ and the Poisson's ratio $v$ are usually calculated from $B$ and $G$ as follows [17]:

$$
E=[9 B G /(3 B+G)] \text {, and } v=(3 B-E) /(6 B),
$$

where $G$ is the shear modulus, which is calculated using the same expressions employed in Ref. [14]. Our results of $G, E$ and $v$ for $\mathrm{ScP}$ material were found to be $67.75 \mathrm{GPa}, 168.3 \mathrm{GPa}$ and 0.24 , respectively. Further, we noted that the values of $G, E$ and $v$ are in well agreement with the theoretical results $(62.18 \mathrm{GPa}$, $156.85 \mathrm{GPa}$ and 0.23 , respectively) reported in Ref. [14]. The Poisson's ratio $v$ is often used to descript the ductile-brittle nature of materials. If the value of $v>0.26$, this implies the ductile behavior of the material, while if $v<0.26$, thus signifies the brittle nature [17]. We can see that the value of $v$ is found to be lower than 0.26 implying that $\mathrm{ScP}$ compound behaves in a brittle manner. 
The hardness $H$ of solid is strongly infected with lattice defects (impurities and dislocations). When considering the dislocations, we find that the hardness $H$ reduces strongly with decreasing dislocation concentration [17]. The Vickers hardness $H_{V}$ can be calculated as follows [18]:

$$
H \mathrm{~V}=1.887 k^{2} G^{0.58},
$$

where $k$ is the Pugh's ratio $(k=G / B)$. Our result of $H_{V}$ for ScP material was found to be $12.7 \mathrm{GPa}$. It is slightly higher than the value (12 GPa) of the silicium (Si) compound [19].

For several covalent and ionic materials (semiconductors, etc.), the fracture toughness $K_{I C}$ can be estimated from the following empirical model [19]:

$$
K_{\mathrm{IC}}=V_{0}{ }^{1 / 6}(B / G)^{1 / 2} G,
$$

where $V_{0}$ is the atomic volume, it is expressed in $\mathrm{m}^{3}$. Both $B$ and $G$ are expressed in MPa. Experimental determination of fracture toughness of solids can be affected by many factors [19]. The fracture toughness $K_{I C}$ of $\mathrm{ScP}$ material was estimated at around $1.39 \mathrm{MPa} \cdot \mathrm{m}^{1 / 2}$. Since the fracture toughness $K_{I C}$ correlates with $H_{V}$ in several III-V compounds [19], we can also clearly observe that our obtained value of $1.39 \mathrm{MPa} \cdot \mathrm{m}^{1 / 2}$ of $K_{I C}$ is near to the value $1.33 \mathrm{MPa} \cdot \mathrm{m}^{1 / 2}$ of $K_{I C}$ for Si material $\left(H_{V}=12 \mathrm{GPa}\right)$ [19].

To the best of our knowledge, the fracture toughness $K_{I C}$ of $\mathrm{ScP}$ not exists in the literature. So, our result is prediction and still await an experimental or other theoretical confirmations.

\subsection{Debye Temperature and Melting Point}

According to Debye's approach, the Debye temperature $\theta_{D}$ can be obtained as follows [17]:

$$
\theta_{D}=\left(\hbar / k_{B}\right)\left(6 \pi^{2} N K / V\right)^{1 / 3} v_{m},
$$

where $\hbar=h / 2 \pi, h$ is the Planck constant; $N$ is the number of unit cells in the volume $V$ of the crystal, $k_{\mathrm{B}}$ is the Boltzmann constant, $K$ is the number of atoms per unit cell, and $v_{m}$ is the average sound velocity, which is calculated using the same method used in [17]. The longitudinal, transverse and average sound speeds of $\mathrm{ScP}$ compound are equal to 7462,4348 and $4823 \mathrm{~m} / \mathrm{s}$, respectively, while the value of $\theta_{D}$ was found to be at around $551.8 \mathrm{~K}$. In addition, in the case of an ionic lattice containing 2 particles, the Debye temperature $\theta_{D}$ can be calculated using the following equation [20]:

$$
\theta_{D}=\left(\hbar / k_{B}\right)\left(5 r_{0} B / \mu\right)^{1 / 2} .
$$

Here $r_{0}$ is the interionic distance, $\mu$ is the reduced mass, and $B$ is the isothermal bulk modulus. The number 5 applies only to crystals with $\mathrm{NaCl}$ structure; it has a different value for other structures.

By substituting our calculated values of $r_{0}$ and $B$ into Eq. (8), the value of $\theta_{D}$ was found to be $522.1 \mathrm{~K}$. It is slightly lower than the value obtained from Eq. (7). To the best of our knowledge, no data reported in the literature on the Debye temperature for $\mathrm{ScP}$ semiconduct- ing compound to make comparison.

For materials with cubic structures, the melting point $T_{m}$ and the elastic constants are related by: $T_{m}=553+5.91, C_{11} \pm 300$ [17], where the melting point $T_{m}$ is expressed in $\mathrm{K}$, while the elastic constants $C_{i j}$ are expressed in $\mathrm{GPa}$. By substituting our calculated value of the elastic constants $C_{11}$ into the previous equation, the melting point $T_{m}$ of $\mathrm{ScP}$ has been evaluated. The obtained value was around to be $2047 \pm 300 \mathrm{~K}$.

\subsection{Other Thermodynamic Properties}

The Debye's vibrational energy $U_{p h}$, the vibrational free energy $F_{v i b}$, the vibrational entropy $S$, and the constant volume heat capacity $C_{\mathrm{V}}$ at different temperature $T$ of $\mathrm{ScP}$ material are calculated. We listed the contribution to the vibrational energy, and the vibrational free energy from 0 to $800 \mathrm{~K}$ in Fig. 4. At $298 \mathrm{~K}$, the $U_{p h}$ is $16.75 \mathrm{~kJ} / \mathrm{mol}$, while that of the $F_{v i b}$ is found at around $2.37 \mathrm{~kJ} / \mathrm{mol}$. This reveals that at low temperature both vibrational energy and free energy have same value for our material of interest.

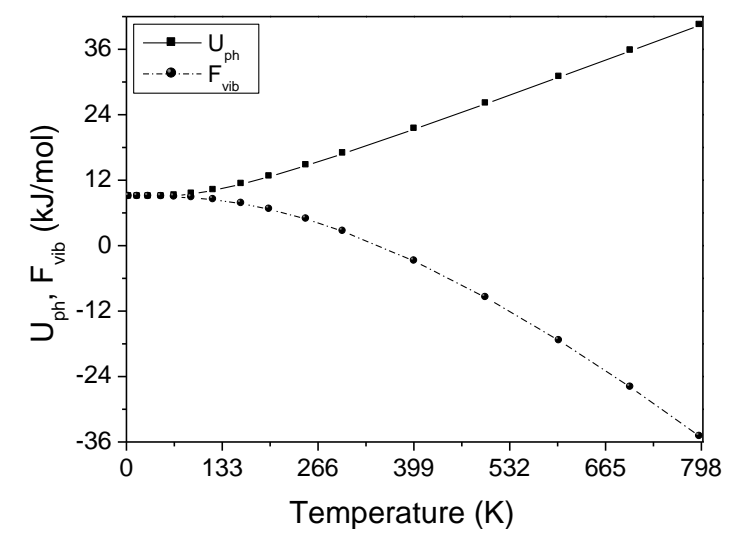

Fig. 4 - Variations of $U_{p h}$ and $F_{v i b}$ of $\mathrm{ScP}$ as a function of $T$

The variations of the vibrational entropy $S$ and the constant volume heat capacity $C_{V}$ with temperature ranging from 0 to $800 \mathrm{~K}$ of $\mathrm{ScP}$ compound are shown in Fig. 5. As the temperature increases, both the entropy $S$ and the heat capacity $C_{V}$ increase as well. At low temperatures, one can observe that $S$ increases quickly with rising the temperature.

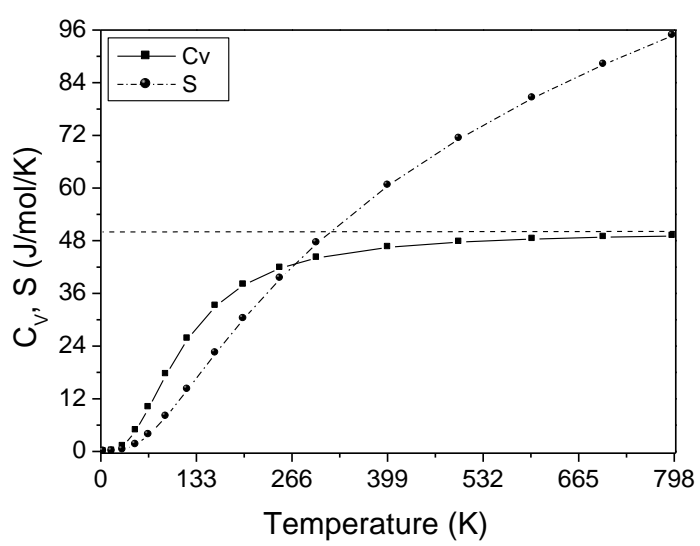

Fig. 5 - Variations of the entropy $S$ and the constant volume heat capacity $C_{V}$ of $\mathrm{ScP}$ as a function of temperature 
It can be seen from Fig. 5 that the $C_{V}$ of $\mathrm{ScP}$ compound increases exponentially with the temperature for $T<580 \mathrm{~K}$, while for high temperature, it approaches the DuLong-Petit limit. It can be noted that similar qualitative behaviors have been reported for $C_{V}$ and $\mathrm{S}$ versus temperature for $\mathrm{MgCa}$ intermetallic compound [17].

\section{CONCLUSIONS}

In the present investigation, we have used the PAW approach in the framework of the DFT as implemented in the Quantum Espresso code. The ground state parameters of both $\mathrm{B} 1$ and $\mathrm{B} 2$ phases of $\mathrm{ScP}$ semiconducting compound are calculated and compared to previous calculations and experimental results. The obtained results of all these parameters are found to be in excellent agreement with the other results available in the

\section{REFERENCES}

1. A. Tebboune, D. Rached, A. Benzair, N. Sekkal, A.H. Belbachir, phys. status solidi $b$ 243, 2788 (2006).

2. E. Parthé, E. Parthé, Acta Cryst. 16, 71 (1963).

3. S. Benalia, M. Merabet, D. Rached, Y. Al-Douri, B. Abidri, R. Khenata, M. Labair, Mater. Sci. Semicond. Process. 31, 493 (2015).

4. W. Celin-Mancera, W. López-Pérez, Á. González-García, L. Ramírez-Montes, R. González-Hernández, Comput. Condens. Matter. 8, 14 (2016).

5. P. Karil, N. Karma, K.K. Choudhary, N. Kaurav, AIP Conf. Proc. 2224, 030001 (2020).

6. S. Baroni, A. Dal Corso, S. Gironcoli, P. Giannozzi, Rev. Med. Phys. 73, 515 (2001).

7. A. Dal Corso, J. Phys. Condens. Matter. 28, 075401 (2016).

8. P.E. Blöchl, Phys. Rev. B 50, 17953 (1994).

9. J.P. Perdew, A. Zunger, Phys. Rev. B 23, 5048 (1981).

10. H.J. Monkhorst, J.D. Pack, Phys. Rev. B 13, 5188 (1976). literature. We also found that under high pressure, $\mathrm{ScP}$ material transforms from B1 to B2 structure at a pressure of around $108 \mathrm{GPa}$ with the corresponding volume collapse $\left(-\Delta V / V_{0}\right)$ of around $4.3 \%$.

Furthermore, the elastic constants, bulk modulus, anisotropy factor, Young modulus, Poisson ratio, hardness $H_{V}$, fracture toughness, longitudinal, transverse and average sound velocities, Debye temperature $\theta_{D}$, and melting point of the B1 phase were also predicted and discussed. The values of $H_{V}$ and $\theta_{D}$ of $\mathrm{ScP}$ were found at around $12.7 \mathrm{GPa}$ and $551.8(522.1) \mathrm{K}$, respectively. To the best of our knowledge, there are no data in the literature on the $H_{V}$ and $\theta_{D}$ of ScP to make comparison. In addition, some other interesting thermodynamic properties as a function of temperature of $\mathrm{ScP}$ material are also discussed in details.

11. S. Daoud, K. Loucif, N. Bioud, N. Lebga, Acta Phys. Pol. A 122, 109 (2012).

12. P. Pandit, B. Rakshit, S.P. Sanyal, phys. status solidi $b$ 248, 921 (2011).

13. A. Maachou, B. Amrani, M. Driz, Physica B 388, 384 (2007).

14. M. Shoaib, G. Murtaza, R. Khenata, M. Farooq, R. Ali, Comput. Mater. Sci. 79, 239 (2013).

15. M. de Jong, W. Chen, T. Angsten, A. Jain, R. Notestine, A. Gamst, M. Sluiter, C.K. Ande, S. Van Der Zwaag, J.J. Plata, C. Toher, S. Curtarolo, G. Ceder, K.A. Persson, M. Asta, Sci. Data 2, 150009 (2015).

16. C.M. Kube, J. Acoust. Soc. Am. 141, 1804 (2017).

17. S. Daoud, N. Bouarissa, A. Benmakhlouf, O. Allaoui, phys. status solidi b 257, 1900537 (2020).

18. X.-Q. Chen, H. Niu, D. Li, Y. Li, Intermetallics 19, 1275 (2011).

19. H. Niu, S. Niu, A.R. Oganov, J. Appl. Phys. 125, 065105 (2019)

20. M. Blackman, Proc. R. Soc. London, Ser. A 181, 58 (1942). 